\title{
Analytic Morphomics Describes Body Composition Associated With Diabetes
}

Olivia Juntila, BS ${ }^{1}$; Jeffrey Friedman, $\mathrm{MS}^{1}$; David Cron, BS ${ }^{1}$; Michael Terjimanian, BS ${ }^{1}$; Michael Englesbe, MD $^{1}$; Stewart Wang, MD, $\mathrm{PhD}^{1}$; Christopher Sonnenday, MD, MHS ${ }^{1}$

\section{ABSTRACT}

\section{Background}

Surgical risk stratification includes demographic factors such as age, body mass index (BMI), and comorbidities. However, the use of BMI is limited in differentiating patients with diabetes mellitus. This study explores the utilization of CT-based images for analytic morphomics to describe body composition differences among patient with and without Type II diabetes mellitus. Measures of abdominal fat, body habitus, and skeletal muscle size were studied as potential descriptors of diabetes status.

\section{Materials and Methods}

This is a retrospective study of trauma patients at the University of Michigan between 2000 and 2013 using CT scans and associated clinical profiles. Visceral anterior body depth (VABD) and lean psoas area (LPA) were measured at the L4 level using established analytic morphomic techniques.
Patients were stratified by gender and BMI weight categories: normal weight $(\mathrm{BMI}<25)$, overweight (25 $\leq \mathrm{BMI}<30)$, and obese (BMI $\geq$ 30). Morphomic measurements were compared within weight categories and multivariate modeling was conducted to determine associations with diabetes.

\section{Results}

Male diabetics consistently had significantly greater VABD and smaller LPA compared to nondiabetics within the same weight category, and VABD and LPA increased as weight category increased. Female diabetics had greater VABD and smaller LPA than nondiabetics within the same weight category, but LPA did not vary significantly among weight categories. In multivariate analysis, VABD, LPA, LPA-age interaction, renal failure, obesity, coronary artery disease, and hypertension were significant 
correlates of diabetes mellitus. The area under the receiver operating characteristic (AUROC) for this model was 0.912 .

\section{Conclusion}

Type II diabetics have greater body habitus and smaller lean trunk muscle mass than nondiabetics. Analytic morphomics offers greater characterization of body composition than BMI. These

\section{Introduction}

Diagnostic use of computed tomography (CT) scans has increased dramatically in the past 2 decades, allowing for faster and more informed medical decision making. Today, however, scan assessment is largely qualitative and represents an untapped resource for quantitative predictors of disease. Analytic morphomics allows for the opportunity to access the plethora of patientspecific information within each scan. It offers increased discrimination between muscle and fat tissue and may serve as a more accurate measure of body composition associated with a chronic disease such as diabetes mellitus. Trunk muscle size, aortic calcification, and fat distribution reflect overall patient health and may offer insight into preliminary body changes related to otherwise unnoticed chronic disease development.

Previous studies have implicated obesity and abdominal fat as strongly associated with clinical diabetes mellitus. ${ }^{1-3}$ Body Mass Index (BMI) has long been used as an index of body composition, providing the basis for weight categories and a tool for overall health risk assessment. However, $\mathrm{BMI}$ is a poor differentiator of muscle and fat and may describe a variety of true body compositions for any given BMI value. ${ }^{4}$ CT-based analytic morphomics have been used to quantify surgical risk associated with body composition in multiple surgical populations. Abdominal aortic calcification is a significant predictor of postoperative morbidity in patients without clinical cardiovascular risk factors. Lower core muscle size (lean psoas area) is strongly measurements could be used to create a diabetic phenotype, and the ubiquity of $\mathrm{CT}$ imaging in modern medicine offers the opportunity for early detection of patients at high risk for development of diabetes mellitus based solely on their body morphomics. Future study is needed to identify prediabetic morphomic phenotypes for early identification and mitigation of adverse health outcomes.

correlated with mortality in major abdominal surgery and liver transplantation., 5,6 These measures may also have utility in describing body-composition associated with higher risk of chronic disease.

Despite recognizing a correlation between Type II diabetes mellitus and body composition changes, the ability to use morphomics to describe chronic health states has not yet been fully explored. ${ }^{7}$ Within this context, this study explores the utilization of CT-based images to describe body composition differences among patients with and without Type II diabetes mellitus. Measures of abdominal fat, body habitus, and skeletal muscle size were studied as potential descriptors of diabetes status.

\section{Methods} Patients

The study population consisted of 2556 adult trauma patients admitted to the University of Michigan Health System (UMHS) between October 2002 and April 2013 with available abdominal CT scans and BMI measurements. Patients were excluded if their CT scans were of poor quality and if their demographic information-namely, height and weight-were not listed in their charts. Only 1169 patients met the inclusion criteria and served as the study cohort. The trauma population offers a cross-sectional sample of the American population and represents a relatively unselected population in terms of prevalence of chronic disease. All patients seen in the UMHS emergency department are included in a prospectively maintained database that uses the standard data definitions and 
collection methods of the National Trauma Data Standard (NTDS). Clinical variables downloaded from this database were supplemented by review of the electronic medical record. Collected variables included height, weight, race, diabetes mellitus, history of smoking, renal failure, bleeding disorder, use of steroids, respiratory disease, dependent functional status, history of alcoholism, existence of an advanced directive, hypertension, congestive heart failure (CHF), coronary artery disease (CAD), cirrhosis, history of cerebrovascular accident (CVA), history of cardiac surgery, and history of myocardial infarction (MI). Diabetes mellitus status was determined by reviewing clinical diagnosis listings in the patients electronic medical record. The study was approved by the University of Michigan Institutional Review Board (HUM 00041441).

\section{Analytic Morphomics}

Analytic morphomics measurements were performed on CT scans in a semiautomated procedure using algorithms programmed in MATLAB v15.0 (MathWorks) that are described in previous studies.8,9 In short, the vertebral levels of the spine were mapped on each CT scan, and crosssectional measurements were taken at the inferior level of the fourth lumbar vertebra. The right and left psoas muscle areas were summed to generate total psoas area. ${ }^{2,8}$ Using radiographic density as measured by Hounsfield units (HU), total psoas area was adjusted to determine the area comprised solely of muscle tissue, referred to as lean psoas area (LPA). ${ }^{9}$ Visceral fat area (VFA) is defined as the cross-sectional area within the fascial envelope with radiographic intensity between -205 and -51 HU. Thresholds were obtained from Mimics software presets (Mimics v14.11, Materialise).${ }^{10}$ In previous studies, the utility of body depth measurements in associations with clinical outcomes and descriptions of body habitus have been examined. ${ }^{11}$ Visceral anterior body depth (VABD), defined as the distance between the anterior face of the $L 4$ vertebra and the anterior fascial envelope, was explored as a descriptor of body habitus. This measure is visually represented in Figure 1.

\section{Weight Categories}

The morphomic profiles of underweight, normal weight, overweight, and obese diabetic and nondiabetic patients were compared. Weight categories were defined as BMI values (in $\mathrm{kg} / \mathrm{m}^{2}$ ): less than $18.5,18.5-25,25-30$, and greater than 30, respectively. ${ }^{12}$ Only 2 patients in the study cohort were defined as underweight and were subsequently dropped from this analysis.

\section{Statistical Analysis}

Descriptive statistics were calculated for the study cohort. Means and standard deviations were used to summarize continuous variables while frequencies and totals were used to summarize categorical variables. Comparisons by diabetes status were computed using Student t-tests or Wilcoxon ranksum tests for continuous variables and Fisher's exact tests and chi-squared tests for categorical variables, where appropriate. LPA, VFA, and anterior body depth variable statistics were displayed by box and whisker plots across gender, diabetes status, and weight categories. Logistic regression was performed to identify factors associated with diagnosis of diabetes. Univariate logistic regres sions were first computed to identify variables appropriate for input into multivariate logistic

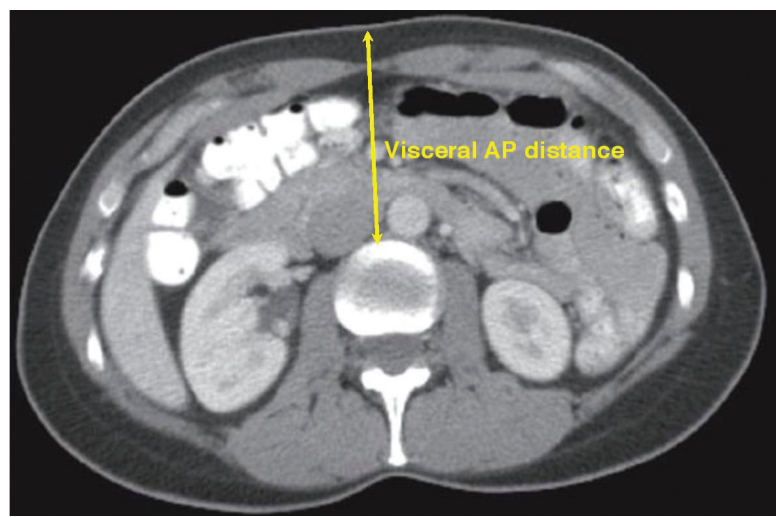

FIGURE 1. Cross-sectional CT image at the L4 vertebral level with demonstration of the visceral anterior body depth measure (yellow arrow). 
regression analysis. Interaction terms between variables were created to test for a nonadditive influence of 2 variables on the model. Selected variables were entered into a multivariate logistic regression in a stepwise backward fashion with an inclusion criterion of $(p<0.10)$. Predictive ability of the model was assessed by the area under the receiver operating characteristic (AUROC) and p-value. A 2-sided significance level of $a=0.05$ was used for all analyses in Statav13.0 (StataCorp).

\section{Results}

1169 patients met inclusion criteria and served as our study cohort. Overall, the mean age of patients included in this analysis was 45.5 years old $(S D=19.6$ years) and mean BMI was 28.1 $\mathrm{kg} / \mathrm{m}^{2}\left(\mathrm{SD}=7.8 \mathrm{~kg} / \mathrm{m}^{2}\right)$. A total of 781 patients (66.8\%) were male, 166 (14.2\%) were nonwhite, and 97 (8.3\%) carried a diagnosis of Type II diabetes mellitus. Of these patients, 737 (63.0\%) presented following a motor vehicle accident, 234 (20.0\%) following a fall, 2 following burn injuries (0.2\%), 9 with a gunshot wound (0.8\%), and 14 (1.2\%) with a stab wound. Descriptive statistics of the total cohort, and of diabetic and nondiabetic patients, are summarized in Table 1. Compared to nondiabetics patients, diabetics were older with higher BMI, more commonly used tobacco, and were more likely to have CHF, CVA, CAD, hypertension, renal failure, a history of cardiac surgery, and a history of myocardial infarction.

TABLE 1. Descriptive Statistics by Diabetic Status and Significance Values

\section{Variable}

Male

\begin{tabular}{lrrr} 
Age (yrs) & $64.8 \pm 13.6$ & $43.8 \pm 19.2$ & $<0.001$ \\
\hline BMI & $33.3 \pm 9.3$ & $27.7 \pm 7.5$ & $<0.001$ \\
Nonwhite race & $14(14.4 \%)$ & $152(14.2 \%)$ & 0.880 \\
\hline Alcoholism & $5(5.2 \%)$ & $118(11.0 \%)$ & 0.083 \\
\hline CHF & $12(12.4 \%)$ & $10(0.9 \%)$ & $<0.001$ \\
\hline History of cardiac surgery & $11(11.3 \%)$ & $30(2.8 \%)$ & $<0.001$ \\
\hline History of myocardial infarction & $15(15.5 \%)$ & $30(2.8 \%)$ & $<0.001$ \\
\hline CVA & $8(8.3 \%)$ & $10(0.9 \%)$ & $<0.001$ \\
\hline CAD & $32(33.0 \%)$ & $42(4.0 \%)$ & $<0.001$ \\
\hline Hypertension & $59(60.8 \%)$ & $188(17.5 \%)$ & $<0.001$ \\
\hline Renal failure & $19(19.6 \%)$ & $24(2.2 \%)$ & $<0.001$ \\
\hline Tobacco use & $10(10.3 \%)$ & $258(24.1 \%)$ & 0.001 \\
\hline Lean psoas area $\left(\mathrm{mm}^{2}\right)$ & $1954 \pm 713$ & $2400 \pm 862$ & $<0.001$ \\
\hline Visceral fat area $\left(\mathrm{mm}^{2}\right)$ & $124 \pm 38$ & $10158 \pm 7995$ & $<0.001$ \\
\hline Visceral AP distance at L4 (mm) & $820 \pm 11635$ & $85 \pm 30$ & $<0.001$ \\
\hline
\end{tabular}

\section{Diabetic $(\mathrm{N}=97) \quad$ Nondiabetic $(\mathrm{N}=1072) \quad$ P-Value}

$62(63.9 \%)$

$719(67.1 \%)$

0.574

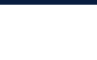


Histograms of LPA showed normal distributions in both diabetics and nondiabetic populations $\left(1954 \pm 713 \mathrm{~mm}^{2}\right.$ vs $2400 \mathrm{~mm}^{2} \pm 862 \mathrm{~mm}^{2}$ $p<0.001$, Figure 2). VFA showed a strong positive skew among both diabetics and nondiabetics $\left(20820 \pm 11635 \mathrm{~mm}^{2}\right.$ vs $10158 \pm 7995$ $\left.\mathrm{mm}^{2}, \mathrm{p}<0.001\right)$. VABD showed a slight positive skew among both diabetics and nondiabetics (124 \pm 38 mm vs $85 \pm 30$ mm, $p<0.001)$. Compared to nondiabetics, diabetic patients had significantly smaller LPA and significantly larger VFA and VABD ( $p<0.001$ for all measurements). LPA was weakly positively correlated with VABD in diabetics and negligibly positively correlated in nondiabetics ( $r=0.229$ and $r=0.091$, respectively). LPA was negligibly correlated with VFA in both diabetics and nondiabetics ( $r=0.074$ and $r=-0.044$ ). VFA and VABD were strongly positively correlated among both diabetics and nondiabetics ( $r=0.816$ and $r=0.813)$.

Box and whisker plots stratified by diabetes mellitus status, gender, and weight categories were generated for LPA, VFA, and VABD (Figure 3). LPA was greater in males than in females and smaller in diabetics than nondiabetics. LPA was greater among nondiabetics than diabetics in both males and females in all weight categories. However, LPA did not vary significantly among female diabetics and nondiabetics across weight categories, and showed a small stepwise increase among male diabetics and nondiabetics across weight categories. VFA and VABD was greater in

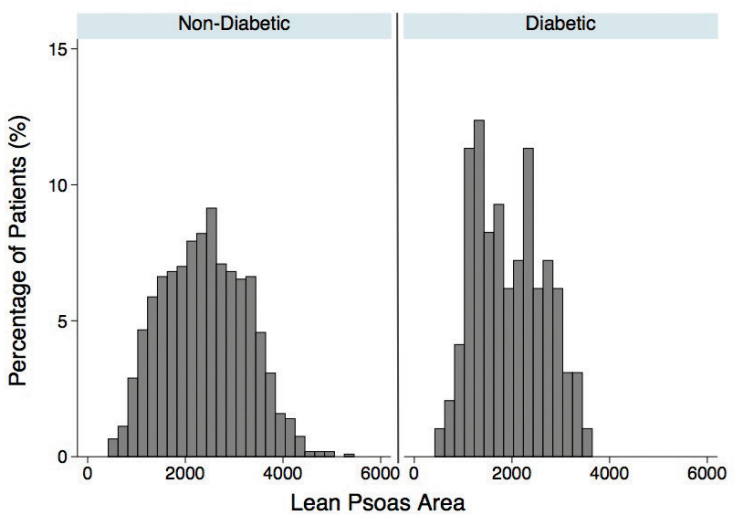

FIGURE 2. Comparison histograms of lean psoas area (LPA) in nondiabetic and diabetic patients. diabetics than in nondiabetics and increased in a stepwise fashion as weight category increased. VFA did not vary between males and females.

In univariate logistic regression, LPA, VFA, and VABD distance were all significant correlates of diabetes (LPA: odds ratio [OR] $=0.52$ per $1000 \mathrm{~mm}^{2}$ increase, 95\% Cl: 0.40-0.68, $p<0.001$; VFA: OR $=1.25$ per $1000 \mathrm{~mm}^{2}$ increase, $95 \% \mathrm{Cl}: 1.17$ 1.28, $p<0.001$; VABD: OR = 1.03 per mm increase, 95\% Cl: 1.03-1.04, $p<0.001$ ). Other significant correlates of diabetes included age, weight category, $\mathrm{CHF}$, and histories of cardiac surgery, myocardial infarction, CVA, CAD, hypertension, renal failure, and tobacco use. In multivariate analysis, VFA fell out of the model due to colinearity with VABD, which remained a strong independent correlate of diabetes $(\mathrm{OR}=1.02,95 \% \mathrm{Cl}: 1.02-1.03, \mathrm{p}<0.001)$. Interaction analysis demonstrated a significant interaction of LPA and age. Upon inclusion of the interaction term into the multivariate model, both LPA and the LPA-age interaction term were significant. Although not significant, gender and age were kept in the model to account for well-described effects on body habitus and development of diabetes. Table 2 shows the estimated parameters for the final model. The AUROC for the complete model was 0.912 .

\section{Discussion}

This study introduces the concept of using analytic morphomics to describe chronic disease states, specifically diabetes mellitus. Diabetics have smaller lean trunk muscle mass, greater visceral fat area, and greater abdomen depth than nondiabetics. Importantly, these analytic morphomic measures appear to differ between patients within the same weight categories and are significant correlates of diabetic status after adjusting for previously described risk factors. Including morphomic measures with common demographic factors resulted in a strongly predictive model of diabetes mellitus presence in an unselected population. This diabetic phenotype and the ubiquity of CT imaging in modern medicine offer the opportunity for early detection of patients at high risk for 

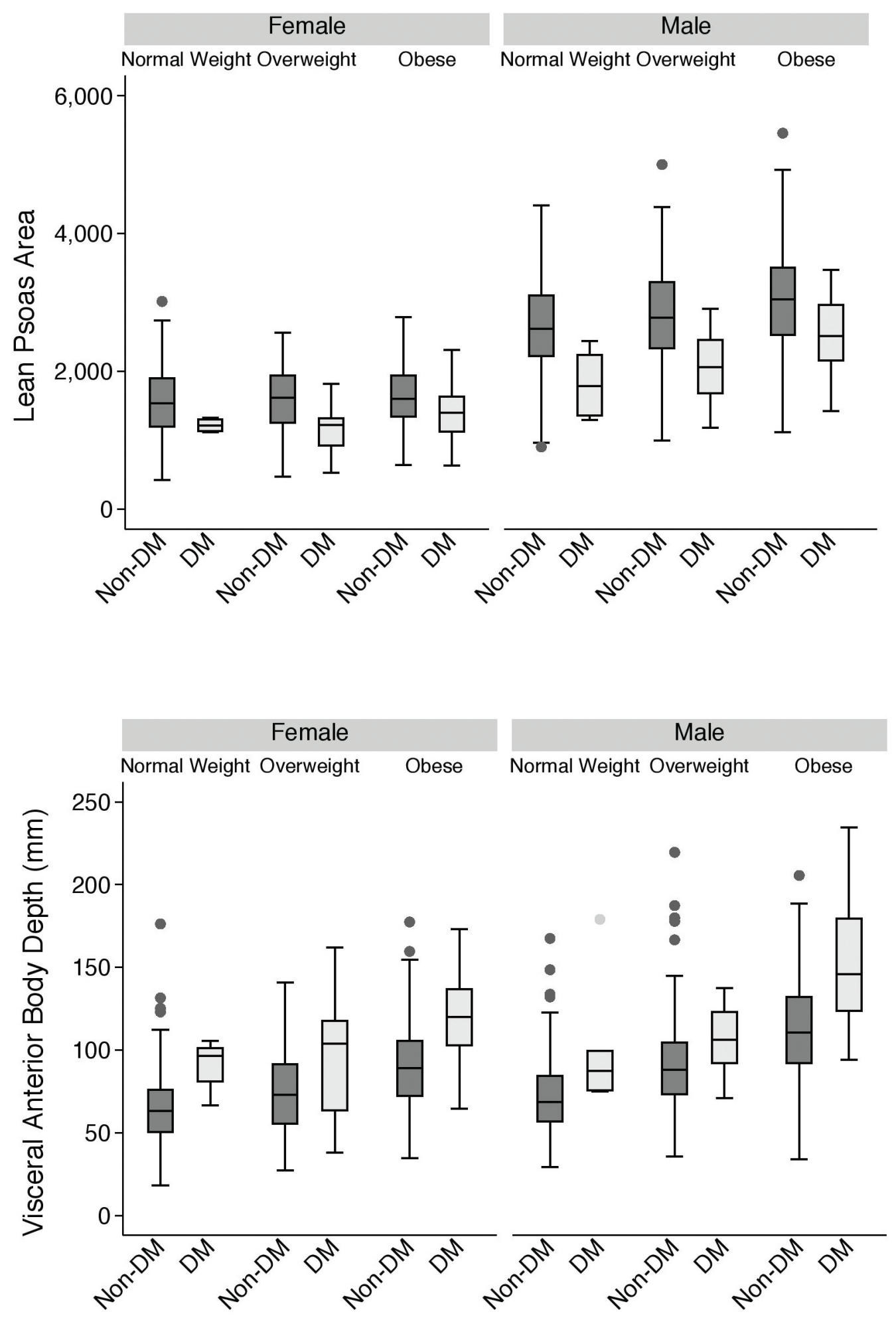

FIGURE 3. Box-and-whisker plots of LPA and VABD. Each measure is displayed by gender, diabetic status, and weight category. 
TABLE 2. Final Multivariate Model Predicting Diabetic Status

\begin{tabular}{lcccc} 
& & & \multicolumn{2}{c}{$95 \%$ Confidence Interval } \\
Variable & OR & P-Value & Lower Limit & Upper Limit \\
\hline Male & 0.795 & 0.578 & 0.355 & 1.781 \\
\hline Age & 0.970 & 0.186 & 0.927 & 1.015 \\
\hline Overweight & 2.168 & 0.064 & 0.956 & 4.916 \\
\hline Obese & 3.952 & 0.002 & 1.683 & 9.280 \\
\hline Hypertension & 2.481 & 0.001 & 1.460 & 4.216 \\
\hline Renal failure & 3.857 & 0.001 & 1.762 & 8.443 \\
\hline Alcoholism & 0.351 & 0.053 & 0.121 & 1.011 \\
\hline CAD & 5.766 & $<0.001$ & 2.872 & 11.575 \\
\hline VABD (mm) & 1.021 & $<0.001$ & 1.012 & 1.030 \\
\hline LPA (per & 0.175 & 0.012 & 0.045 & 0.678 \\
\hline 1000 mm $\left.{ }^{2}\right)$ & & & & 1.049 \\
\hline LPA-age & 1.026 & 0.024 & 1.003 & \\
interaction & & & & \\
\hline & & & & \\
\hline
\end{tabular}

development of diabetes mellitus based solely on their body morphomics. Although laboratory measurements (such as hemoglobin A1C) have already been established for the diagnosis of type II diabetes mellitus, analytic morphomics offers a unique opportunity to obtain more information from cross-sectional imagining without additional invasive testing. These tools could allow for clinical interventions of healthy living in individuals with morphomic measurements consistent with a diabetic phenotype but who have not yet been diagnosed with type II diabetes.

Previous studies have shown that overweight status, especially obesity, substantially increases lifetime risk of diabetes, particularly at younger ages. ${ }^{13}$ Prior cross-sectional studies using combined CT and dual energy $\mathrm{x}$-ray absorptiometry measurements have reported a strong correlation between Type II diabetes mellitus in obese adults and increased visceral and subcutaneous adipose tissue. ${ }^{14}$ Our findings confirm this observation of increased visceral fat in diabetics, and we report increasing sarcopenia in these patients. Traditionally, sarcopenia was thought to be a result of aging; however, increasing evidence shows that sarcopenia is linked to chronic disease states, such as impaired glucose tolerance, and negative surgical outcomes. ${ }^{15}$ Diabetic patients, and chronic disease patients in general, require more care and are more likely to undergo surgery than unburdened patients. In the era of capitated care and patient-centered medicine, early detection and intervention of chronic disease is critical for reducing the burden of advanced cases and improving community health. Analytic morphomics offer improved utilization of CT scan data and greater phenotype discrimination for risk stratification of chronic disease states.

In this sample of trauma patients, analytic morphomics identified phenotypic differences in diabetic patients, independent of BMI category. We 
observed a pattern of increased visceral fat and abdomen depth and decreased lean psoas muscle in patients already carrying a diagnosis of diabetes mellitus. However, the normal course of morphomic changes that occur as individual patients transition from nondiabetic to prediabetic and finally to diabetic status remains unknown. A large prospective study is needed to accurately map these changes in a representative cross-section of the US population. Additionally, a clinical study of obese women by Pasquali et al demonstrated that metformin reduced body weight and BMI as compared to the placebo group..$^{16}$ As such, any future prospective studies of early diabetic detection and phenotype must control for treatment history to determine the effect of diabetes therapies on morphomic characteristics.

This study was performed retrospectively at a single tertiary care center in the Midwest, which may not be representative of the entire US population. The trauma population was chosen to represent a relatively normal population; however, as is common in the trauma population, young and male patients were overrepresented. Within this population, patients without abdominal CT scans were excluded, which may bias this investigation toward specific patients or injury patterns. Finally, patient history details were queried from the trauma registry, which uses NTDS definitions and does not include variables such as time of diagnosis, treatment details, or $\mathrm{HbA} 1 \mathrm{c}$ lab results. Additionally, intrinsic to being a trauma patient in a tertiary care center, these patient's charts may not be thoroughly correct or complete. Regardless, this study demonstrates the rich data source available in commonly used CT scans and the ability to correlate morphomic measures with a chronic disease diagnosis.

\section{Conclusion}

This study demonstrated that diabetics have smaller lean trunk muscle mass and greater abdominal size than nondiabetics, as determined by analytic morphomics. Analytic morphomics offer greater characterization of body composition than BMI. Future study is needed to identify additional morphomic phenotypes associated with chronic disease and adverse health outcomes, as well as determine the independent effect that morphomic characteristics may have on health outcomes.

\section{Acknowledgments}

The authors thank Mirabelle Lindquist, Ali Hammoud, Mitchell Alameddine, and Jake Claflin for their work in processing CT scans for analysis in this work.

\section{References}

1. Koh-Banerjee P, Wang Y, Hu FB, Spiegelman D, Willett WC, Rimm EB. Changes in body weight and body fat distribution as risk factors for clinical diabetes in US men. Am J of Epidemiol. 2004;159(12):1150-1159.

2. Carey VJ, Walters EE, Colditz GA, et al. Body fat distribution and risk of non-insulin-dependent diabetes mellitus in women: the nurses' health study. Am J of Epidemiol. 1997;145(7):614-619.

3. Chan JM, Rimm EB, Colditz GA, Stampfer MJ, Willett WC. Obesity, fat distribution, and weight gain as risk factors for clinical diabetes in men. Diabetes Care. 1994;17(9):961-969.

4. Baumgartner RN, Heymsfield SB, Roche AF. Human body composition and the epidemiology of chronic disease. Obesity Research. 1995;3(1):73-95.

5. Harbaugh CM, Terjimanian MN, Lee JS, et al. Abdominal aortic calcification and surgical outcomes in patients with no known cardiovascular risk factors. Ann Surg. 2013;257(4):774-781.

6. Englesbe MJ, Patel SP, He K, et al. Sarcopenia and mortality after liver transplantation. J Am Coll Surg. 2010;211(2):271-278.

7. Park SW, Goodpaster BH, Lee JS, et al. Excessive loss of skeletal muscle mass in older adults with type 2 diabetes. Diabetes Care. 2009;32(11):1993-1997.

8. Lee JS-J, He K, Harbaugh CM, et al. Frailty, core muscle size, and mortality in patients undergoing open abdominal aortic aneurysm repair. J Vasc Surg. 2011;53(4):912-917.

9. Englesbe MJ, LeeJS, He K, et al. Analytic morphomics, core muscle size, and surgical outcomes. Ann surg. 2012;256(2):255-261. 
10. Parenteau CS, Zhang P, Holcombe S, KohoydaInglis C, Wang SC. Can anatomical morphomic variables help predict abdominal injury rates in frontal vehicle crashes? Traff Inj Prev. 2013;15(6):619-626.

11. Lee JS, Terjimanian MN, Tishberg LM, et al. Surgical site infection and analytic morphometric assessment of body composition in patients undergoing midline laparotomy. J Am Coll Surg. 2011;213(2):236-244.

12. National Heart, Lung, and Blood Institute. Panel NHLBI Obesity Education Initiative Evaluation. Clinical Guidelines on the Identification, Evaluation, and Treatment of Overweight and Obesity in Adults. Bethesda (MD); 1998.

13. Narayan KV, Boyle JP, Thompson TJ, Gregg EW, Williamson DF. Effect of BMI on lifetime risk for diabetes in the US. Diabetes Care. 2007;30(6):1562-1566.
14. Smith SR, Lovejoy JC, Greenway F, et al. Contributions of total body fat, abdominal subcutaneous adipose tissue compartments, and visceral adipose tissue to the metabolic complications of obesity. Metabolism. 2001;50(4):425-435.

15. Sayer AA, Dennison EM, Syddall HE, Gilbody HJ, Phillips DI, Cooper C. Type 2 Diabetes, muscle strength, and impaired physical function: the tip of the iceberg? Diabetes Care. 2005;28(10):2541-2542.

16. Pasquali R, Gambineri A, Biscotti D, et al. Effect of long-term treatment with metformin added to hypocaloric diet on body composition, fat distribution, and androgen and insulin levels in abdominally obese women with and without the polycystic ovary syndrome. J Clin Endocrinol Metab. 2000;85(8):2767-2774. 